\title{
Screening for mutation site on the type I neurofibromatosis gene in a family
}

\author{
Ming Lv • Wenhua Zhao $\cdot$ Lin Yan • Liang Chen • \\ Kai Cui $\cdot$ Jie Gao $\cdot$ Fachang Yu $\cdot$ Sheng Li
}

Received: 20 November 2011 / Accepted: 28 November 2011 /Published online: 30 December 2011

(C) The Author(s) 2011. This article is published with open access at Springerlink.com

\begin{abstract}
Purpose The purpose of the study was to determine the sites and types of mutations associated with type I neurofibromatosis (NF1) in the NF1 gene in a family with NF1 patients. Methods The blood samples obtained from this family (four patients and one normal healthy individual) were analyzed
\end{abstract}

Ming Lv and Wenhua Zhao contributed equally to this work.

M. Lv

Department of Orthopedics, Central Hospital of Zibo City,

Zibo 255000, China

W. Zhao

Department of Oncology,

Qianfoshan Hospital of Shandong Province,

Jinan 250014, China

S. Li $(\bowtie)$

Department of Hepatobiliary Surgery, Shandong Cancer Hospital, Jinan 250117, China

e-mail: Drlisheng@sohu.com

L. Yan

The No.148 Hospital of People's Liberation Army,

Zibo 255300, China

L. Chen

Department of Orthopedics, Shandong Provincial Hospital,

Jinan 250021, China

K. Cui

Key Laboratory for Rare and Uncommon

Diseases of Shandong Province,

Jinan 250117, China

J. Gao $\cdot$ F. Yu

Academy of Medicine and Life Science,

Shandong Academy of Medical Sciences,

Jinan 250012, China by performing polymerase chain reaction (PCR) and DNA sequencing for mutation screening.

Results We found synonymous mutations in exons 7, 38, 50 , and 56 of the NF1 gene. This implied that the third codon had a new SNP that did not lead to a change in the amino acid coding. The exon 19 mutation was CAG homozygous, while it was C/TAG heterozygous in normal individuals. The stop codon led to nonsense-codon-mediated decay of the mRNA (NMD), thus resulting in only one copy of the NF1 gene that encodes the normal protein in individuals.

Conclusions The synonymous mutations in the NF1 gene occur in exons $7,38,50$, and 56 . The CAG homozygous mutations may occur in exon 19, and the C/TAG heterozygous mutations may occur in the others. This mutation may be responsible for NF1 in patients in this family and may warrant extensive research on the $N F 1$ gene.

Keywords Gene · Neurofibromatosis · Hereditary diseases . Cafe au lait spots $\cdot$ Mutation $\cdot$ Exon

\section{Introduction}

Neurofibromatosis (NF) is a common dominant genetic disease that is caused by an aberration in a dominant gene and manifests as tumors of neuroectodermal and mesodermal origin. It is characterized by multi-system and multiorgan involvement, especially involvement of the central nervous system, and a variety of tumors. Type I NF, also known as the Von Recklinghausen disease, is the most common type. The gene located on chromosome 17q11.2 is the main cause of multiple nervous system tumors characterized by pigmented skin lesions and multiple NF; it may also be associated with mental retardation, neurological 
changes, and neurofibroma-like and sarcoma-like changes. NF1 is currently reported to be associated with a total of 728 mutations, including base substitutions, major mutations, insertions, deletions, nonsense mutations, missense mutations, and frameshift mutations. Most mutations can lead to the production of truncated proteins. By using polymerase chain reaction (PCR) and DNA sequencing, we screened members of a Chinese family for NF gene mutations and found that four members had NF1 and one was normal and healthy.

\section{Materials and methods}

\section{Clinical data}

We collected the dossiers on a family with patients having type I NF from the Shandong Cancer Hospital between 2009 and 2010. We had data on eight people in the family, of whom six were diagnosed with type I NF. All the patients were diagnosed on the basis of the US NIH diagnostic standard of the year 1987.

The clinical data of three generations of the family has been shown in Fig. 1. The proband (III1) was a 21-year-old woman who had undergone a surgery at the age of 9 years because of a swelling and pain in her left lower extremity along with coffee-with-milk spots (Fig. 2); the pathological diagnosis was NF (Fig. 3). Case I 1 was of a 69-year-old man who had lesions on his abdominal wall, arms, angle of the eyes, and other sites (Fig. 4), and case I 2 was of a healthy female family member. Case II 1 was of a 36-yearold woman with a 1-year history of NF, and case II 2 was of a 40-year-old man with dorsal neurofibroma (Fig. 5). Case II 1 was of a 40-year-old woman who was a healthy subject, and case II 2 was of a 7-year-old girl showing coffee-withmilk spots; she did not have NF. Case III 3 was of a 1 -year-old female child with coffee-with-milk spots and no NF.

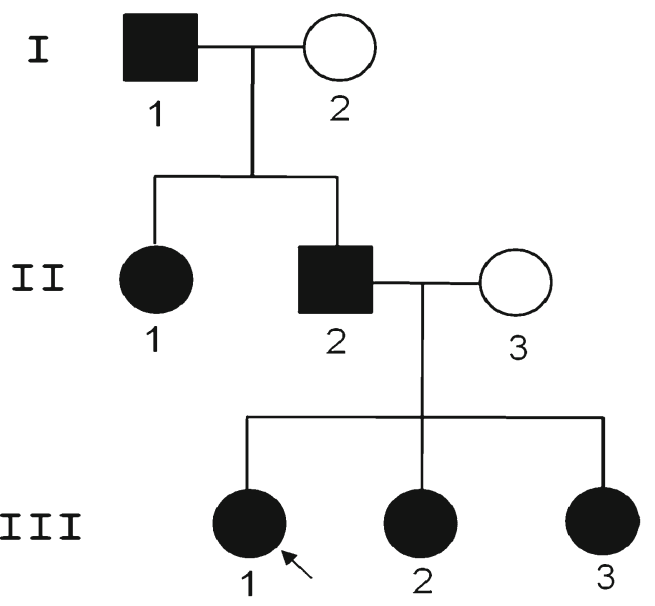

Fig. 1 A diagram showing the affected members of the family

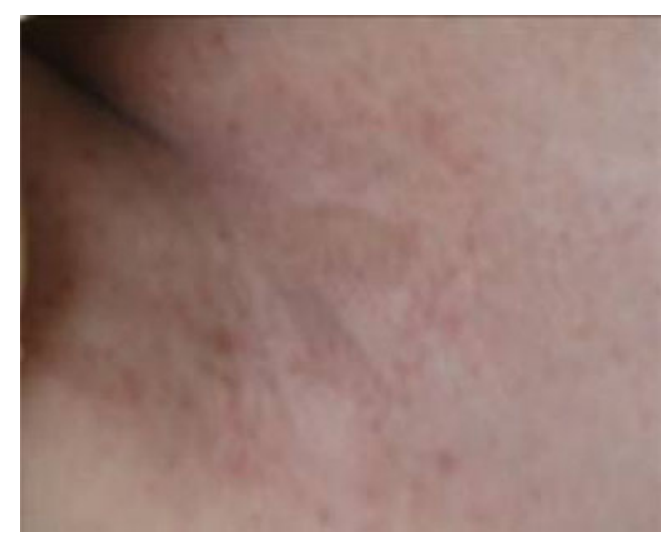

Fig. 2 Patient III 1. A diagram showing the affected members of the family axillary coffee-with-milk spots

\section{DNA extraction}

Our study was approved by the medical ethics committee of the hospital, and we obtained informed consents from the family members before collecting peripheral blood samples from four patients diagnosed with NF (I 1, II 1, II 2, III 1) and one normal healthy subject (II 3 ) of the same family. We stored the blood samples at $-85^{\circ} \mathrm{C}$ in an ultra-low temperature freezer. We extracted the $N F 1$ gene DNA by using a modified salting-out and precipitation techniques and measured the DNA concentration by using a spectrophotometer.

\section{Primer design}

We used the NCBI database to obtain the NF1 salting-out and precipitation techniques salting-out and precipitation techniques gene sequence salting-out and precipitation techniques and the Primer 5.0 software for designing the primers required to amplify the region coding for the $N F 1$ gene (Table 1).

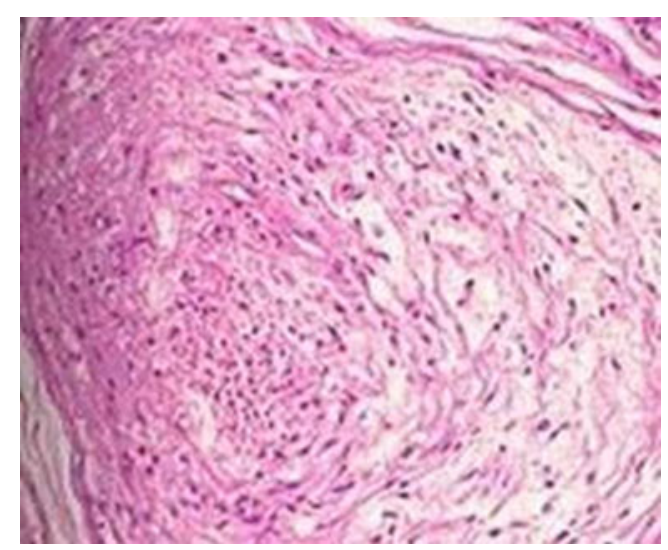

Fig. 3 Patient III 1. Postoperative pathological examination 




Fig. 4 Patient I 1. Neurofibroma on the anterior chest

\section{PCR amplification}

The PCR reaction setup has been shown in Table 2. This reaction was set up in the Biometra PCR instrument, and the reaction conditions were as follows: polymerization at $95^{\circ} \mathrm{C}$ for $5 \mathrm{~min}$, followed by cooling in an ice bath, a quick spin, addition of $1 \mu \mathrm{l} \mathrm{Taq}$ DNA polymerase, and a final quick spin for mixing. The thermal cycling amplification conditions were as follows: pre-denaturation reaction at $96^{\circ} \mathrm{C}$ for $3 \mathrm{~min}$, denaturation at $96^{\circ} \mathrm{C}$ for $30 \mathrm{~s}$, annealing at $55^{\circ} \mathrm{C}$ for $55 \mathrm{~s}$, extension at $72^{\circ} \mathrm{C}$ for $30 \mathrm{~s}$, and lastly a final extension at $72^{\circ} \mathrm{C}$ for $10 \mathrm{~min}$. The PCR amplification products were analyzed by performing agarose gel electrophoresis. We used Exon I and the shrimp alkaline phosphatase enzyme for purification.

\section{DNA sequencing}

DNA was sequenced by DNA dideoxy sequencing method by using Biomek FX Beckman fluid moving workstation and a NanoDrop 8000 spectrophotometer. We then transferred the reaction plate to the Applied Biosystems $3730 \mathrm{XL}$

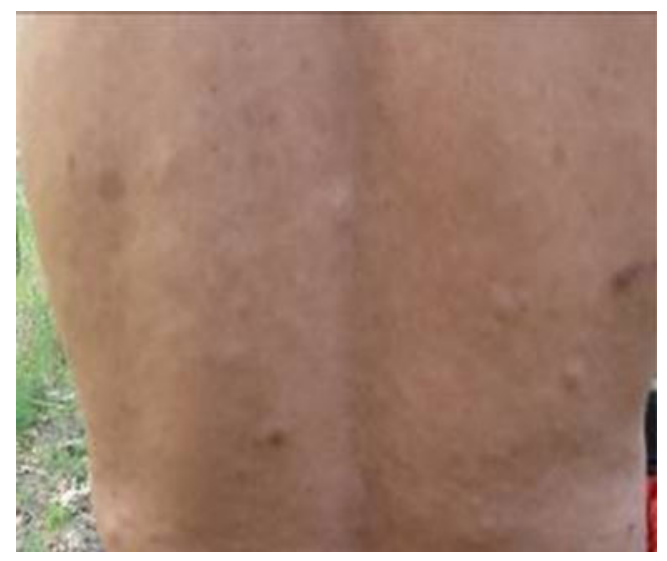

Fig. 5 Patient III 2. Dorsal neurofibroma surgery fully automatic DNA sequencing instrument and electrophoresed the samples. When we obtained abnormal DNA sequencing results, we used the TA cloning system kit (Invitrogen, San Diego, CA) and directly sequenced the PCR products; we then constructed a color map representing the DNA sequence.

\section{Results}

We detected $\mathrm{NF1}$ gene mutations in five blood samples from this family by direct sequencing and DNA cloning methods, and we found totally synonymous mutations in four NFI exons - exon 7, exon 38, exon 50, and exon 56. These mutations were new single-nucleotide polymorphisms (SNPs), located on the third codon position, and the amino acid coding did not change. We used a combination of specific primers and universal primers for sequencing, but the peaks were disordered, which indicated that this part of the exon possibly had an indel mutation and should be sequenced. On comparison of the forward and reverse sequencing results, we observed that this part of the exon had a more complex structure and had both an SNP and a deletion mutation, which should be sequenced.

Cloning and sequencing of exons 18, 19, 20, 22, 23, 26, 27 , and 36 showed that these exons were completely normal and that there was a nonsense mutation in exon 19 (CAG [glutamine] $>$ TAG [stop codon]) (see Fig. 6). The normal healthy individual was a CAG homozygote, and the patients were C/TAG heterozygotes. The stop codon led to nonsense-codon-mediated mRNA decay (NMD), which led to the formation of only one copy of the NF1 gene that encodes the normal protein in individuals.

\section{Discussion}

$\mathrm{NF}$ is a common autosomal dominant genetic disease manifested as neuroectodermal abnormalities. There are approximately 15 million NF patients worldwide with a clear family history, often involving multiple systems and organs [3]. In Von Recklinghausen disease (or NF1-type), the aberrant gene is located on human chromosome 17q11.2 and has a length of $350 \mathrm{~kb}$, with 60 exons; the transcription length is about $11-13 \mathrm{~kb}$, also a $315-\mathrm{kb}$ region of chromosome that is composed of three untranslated regions. Kang et al. [4] found that specific methylation can occur at the transcription-factor-specific binding sites on the NF1 gene promoter, which is parallel and conformal to tumor cell genome hypomethylation.

NF1 gene mutations mainly include DNA replication errors, point mutation, and other mutations, with replication errors accounting for $20 \%$ to $30 \%$ gene mutations. The 
Table 1 Primer sequences of the target gene

\begin{tabular}{|c|c|c|}
\hline Exons & Primer sequence $\left(5^{\prime} \rightarrow 3^{\prime}\right)$ & PCR product length $(\mathrm{bp})$ \\
\hline \multirow[t]{2}{*}{ E1 } & GAGCCTGCACTCCACAGACCC & 297 \\
\hline & TCCTCCAGAGCCTGAGGCAGC & \\
\hline \multirow[t]{2}{*}{ E 2} & ATGGCAAGTAAGTTATTTATGGTC & 331 \\
\hline & TATCCAAAGTCCACAGAAAATCA & \\
\hline \multirow[t]{2}{*}{ E 3} & GAGGTAAAATGGAAGACTATTGTTG & 363 \\
\hline & AAAATTGTCTGTCACCAGGTCAG & \\
\hline \multirow[t]{2}{*}{ E 4} & TGGTAAGGATGGCAGGGGGATTG & 465 \\
\hline & TCCCATGTGGATTTACACACTAACC & \\
\hline \multirow[t]{2}{*}{ E5 } & GATGTCTTGCTATGTTGCCCAGG & 479 \\
\hline & ATTGCCAAGATTTAAAATGCTCA & \\
\hline \multirow[t]{2}{*}{ E6-7 } & AATCATTGATGTCCAAGGCATAT & 723 \\
\hline & TTTCCTATTTGACACCAGTTGAC & \\
\hline \multirow[t]{2}{*}{ E8 } & CCAGGGATTTTGTTCCTATCTAA & 483 \\
\hline & CAGAGGTATCGTTTAGTCTTCTCAGA & \\
\hline \multirow[t]{2}{*}{ E9 } & TTCTTTATAGTATGAGTTTTAGAGGC & 402 \\
\hline & GAACTTATCAACGAAGAGTCAGA & \\
\hline \multirow[t]{2}{*}{ E10-11 } & GGGTGCTTTGTGCTTCTTCTGGC & 651 \\
\hline & AAGAAATACGCAAAGAAAAGAAAGA & \\
\hline \multirow[t]{2}{*}{ E12 } & TGGAAATCATGGTGTGTGTTTGC & 361 \\
\hline & AAAATAACCAAAGCAGCAGGAAT & \\
\hline \multirow[t]{2}{*}{ E13 } & AATACTGACCTTATGCTTACTATTGA & 384 \\
\hline & TATCCTCAAGGTCTTGGCGTTTC & \\
\hline \multirow[t]{2}{*}{ E14 } & TTGAAGTTTCCTTTTTTTCCTTGCA & 221 \\
\hline & AAACCACACACCAAAGGAACATCAT & \\
\hline \multirow[t]{2}{*}{ E15 } & AATGAAAGAGCTCAATTTCTTAGCA & 315 \\
\hline & AAACCATAAAACCTTTGGAAGTGTA & \\
\hline \multirow[t]{2}{*}{ E16 } & CTCTAAACTTGTATTCATTATGGGAGA & 288 \\
\hline & ATCTCTCACCATTACCATTCCAA & \\
\hline \multirow[t]{2}{*}{ E17 } & ATTCCTCTTGGTTGTCAGTGCTT & 260 \\
\hline & AACAAACAGAGCACATAAAATGATACA & \\
\hline \multirow[t]{2}{*}{ E18 } & TTATACATAAAATTACCCAAGTTGCA & 337 \\
\hline & ACTGAGTAAAAAAAACCACTATTCACA & \\
\hline \multirow[t]{2}{*}{ E19-20 } & TGGCAGGCAGGGCTCTAAGTG & 473 \\
\hline & TAACAGACAAAAGTCAACTTTACAGA & \\
\hline \multirow[t]{2}{*}{ E21 } & TAAGGTTTAATTCATGCTTTGCA & 516 \\
\hline & ATAGAGAAAGGTGAAAAATAAGAGAAC & \\
\hline \multirow[t]{2}{*}{ E22-23 } & CTCTAGGGGGTCTGTCTTCTGGG & 518 \\
\hline & TGGACCATATTCCCAAGCACACG & \\
\hline \multirow[t]{2}{*}{ E24 } & TGTCACTTAGGTTATCTGGCAAA & 453 \\
\hline & CTGTAGACTATTCTTCATAAACTGACAAC & \\
\hline \multirow[t]{2}{*}{ E25 } & TTTAAGGTAGCCATTTTGCCAAGAT & 400 \\
\hline & TTGCTTTATGTTTTTTGGTGACT & \\
\hline \multirow[t]{2}{*}{ E26-27 } & TTGTTTTGGAATGTCTGGTTAGC & 622 \\
\hline & ACTTTTCTTCCCCGCTTACTCTA & \\
\hline \multirow[t]{2}{*}{ E28-29 } & TTCCTACCTAAGAATAAAAATGGGA & 502 \\
\hline & AACAGCGGTTCTATGTGAAAAGAT & \\
\hline E30 & CGTAAGCCATCCAGCCCTGTCAA & 460 \\
\hline & CAATTCTCAATGTATTATTCATCCAAAC & \\
\hline E31 & TGTTGCTGTATGTAGTCGGTGCT & 226 \\
\hline
\end{tabular}


Table 1 (continued)

\begin{tabular}{|c|c|c|}
\hline Exons & Primer sequence $\left(5^{\prime} \rightarrow 3^{\prime}\right)$ & PCR product length (bp) \\
\hline & TTTACAGTGAAGGTCAAATAGGC & \\
\hline \multirow[t]{2}{*}{ E32 } & GGACTGATTGATTCAGAGTTTTTATG & 389 \\
\hline & GCACATAACTGAAAACCATAGGG & \\
\hline \multirow[t]{2}{*}{ E33 } & TATTTGGGAAGGTTAGAAACACT & 305 \\
\hline & GAGCAACTGAGTAAGTGGCAAGA & \\
\hline \multirow[t]{2}{*}{ E34 } & ATAAGTCTGGGTGTATCTGGTGT & 459 \\
\hline & AAAGAGCAAATCTGTGATTTCTT & \\
\hline \multirow[t]{2}{*}{ E35 } & GGGAAAAGTAGTGGACTGTGAAGC & 452 \\
\hline & GTGGCAAACTCTCCTTCTCAACC & \\
\hline \multirow[t]{2}{*}{ E36 } & TACCCTTTAGAATGCCTGTTGCT & 318 \\
\hline & AACTTGCCATCTCTCTATATTTGCTA & \\
\hline \multirow[t]{2}{*}{ E37 } & ATTCCCACTGTTTTCTTCCTTTC & 776 \\
\hline & GAGGCCAGGATATAGTCTAGTTAGTCA & \\
\hline \multirow[t]{2}{*}{ E38 } & ATTCTTCTCCACTTCACCCCGTC & 417 \\
\hline & ACCCCAAATCAAACTGAAGAGAA & \\
\hline \multirow[t]{2}{*}{ E39 } & AAGGGGTATTTTGGTTTTACTGTAG & 622 \\
\hline & TAAAGTACCAAACTCTTGCCGCT & \\
\hline \multirow[t]{2}{*}{ E40 } & CAGGCCTGATTCTAGGTAATAGTCTT & 432 \\
\hline & ACTGTGTTTTTTACAACTCTATCCCTA & \\
\hline \multirow[t]{2}{*}{ E41-42 } & АTCTCTTAATCTCTGAAGGAGTCAA & 865 \\
\hline & TCAGGTGAAGTAAAATGGAGAAA & \\
\hline \multirow[t]{2}{*}{ E43 } & AATACTCAGTGCCAGTTGACCAT & 676 \\
\hline & ATGCCTAAAAAAGGGGATACTCT & \\
\hline \multirow[t]{2}{*}{ E44-45 } & CAATATGTATTCAGAGTATCCCCTTT & 384 \\
\hline & ATATTTGGGAGAAGTGAGGGCGG & \\
\hline \multirow[t]{2}{*}{ E46 } & TCCGAGATTCAGTTTAGGAGTTA & 342 \\
\hline & CTAATATGACTACTTCAAACAACTAAA & \\
\hline \multirow[t]{2}{*}{ E47 } & GAAGAATCAACAAACCTTGGTGA & 480 \\
\hline & GCAACAAGAAAAGATGGAAGAGTAC & \\
\hline \multirow[t]{2}{*}{ E48 } & AAGAAAGCTACTGTGTGAACCTCAT & 511 \\
\hline & GCTCAAGCAATCCTTCCATCTAT & \\
\hline \multirow[t]{2}{*}{ E49 } & GTCAGGGAAGAAGACCTCAGCAG & 333 \\
\hline & ACTGTGAACTTTCTGCTCTGCCA & \\
\hline \multirow[t]{2}{*}{ E50 } & GTTTCTCTACTCAGCAACACTTAGC & 754 \\
\hline & AGCACAATCAGACTGGAAGAATAA & \\
\hline \multirow[t]{2}{*}{ E51 } & ACTTGGAAGGAGCAAACGATGGT & 496 \\
\hline & AGCAAAGGCAAAACAAAATAAGG & \\
\hline \multirow[t]{2}{*}{ E52 } & TTAAACACTTTATGTCCAAACATTT & 437 \\
\hline & TGGCTACCTATTTACAATGCTGT & \\
\hline \multirow[t]{2}{*}{ E53-54 } & GGTGAAGTGATTATCCAGGTGTT & 515 \\
\hline & TTAACTTAAAGACAGGCACGAAG & \\
\hline \multirow[t]{2}{*}{ E55-56 } & AATGAAGAAATGCCCCAGAAAG & 769 \\
\hline & CATTGTGTGTTCTTAAAGCAGGC & \\
\hline \multirow[t]{2}{*}{ E57 } & TATTTTTGGCTTCAGATGGGGAT & 439 \\
\hline & AAAGTCAAGTCAGTTACAAGGTA & \\
\hline \multirow[t]{2}{*}{ E58 } & AATGTGTCCCCGTTGTTAAGCGA & 310 \\
\hline & GGGCAAGGACAGGGAAGGGG & \\
\hline
\end{tabular}


Table 2 PCR reaction system setup

PCR reaction system

Volume $(\mu \mathrm{l})$

Phusion ${ }^{\circledR}$ high-fidelity DNA polymerase

0.5

Phusion ${ }^{\circledR}$ HF buffer pack $(5 \times)$

10

Deoxynucleotide solution mix (10 mM)

DMSO (100\%)

1

F primer $(2.5 \mu \mathrm{M})$

R primer $(2.5 \mu \mathrm{M})$

DNA template

$\mathrm{H}_{2} \mathrm{O}$

Total volume

addition or deletion of a small direct repeat sequence in the open reading frame can result in deletion of a terminal inverted repeat sequence during replication, or when skipped reading phenomenon in gene sequence occurs and DNA hairpin-like structure is formed, which eventually leads to the deletions of some DNA sequences. The mechanism underlying these two mutations - in which a single-base substitution results in a missense or a nonsense mutation known as gene point mutation - is fairly complex and unclear. The point

mutations are missense mutations with an incidence of about $5-10 \%$, and nonsense mutations have an incidence of approximately $30-38 \%$. The incidence of missense mutations or nonsense mutations that may affect RNA splicing is $20 \%$ to $35 \%$. Our study showed that synonymous mutations can occur in the following exons in NF1: exon 7, exon 38, exon 50, and exon 56. Thomson et al. [9] reported the first new exon mutation in exon 26 of the NF1 gene (A4435G, Ser1479Gly). Analysis of exons 24 to 29 showed that a new mutation in exon 26 affecting RNA splicing resulted in skipped reading phenomenon of the first 68 amino acids in exon 26, which caused a reading frameshift mutation, as analyzed by RT-PCR technique. Messiaen et al. [6] found that a mutation (A1466G) in the region of exon 10 also had a dormant splicing receptor, which can lead to frameshift mutations.

The effect of mutations on gene transcriptional levels has been reported in recent years $[1,7]$. The rates of occurrence of other mutations, including large gene-fragment insertions and large gene-fragment deletions, are less than 5\%. Chromosomal translocation and inversion are rare types of mutations. The rate of occurrence of mutations caused by large gene-fragment deletions, covering the whole $N F 1$ gene and the neighboring genes, is about $2 \%$ to $10 \%$, and it mainly

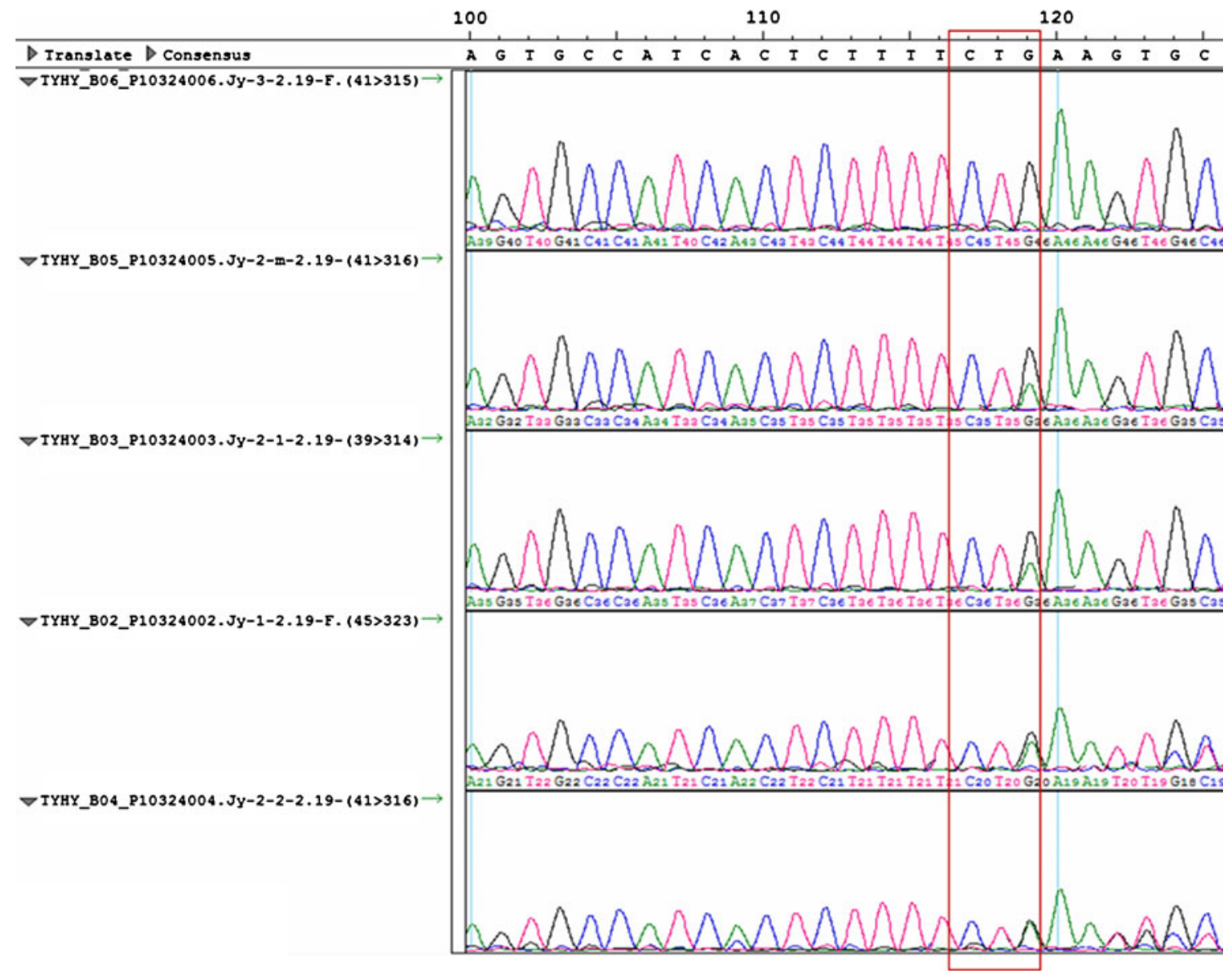

Fig. 6 Exon 19 
leads to a deletion through simple sequence repeats $[2,8]$. Studies confirmed that deletions of $112 \mathrm{Mb}$ and $114 \mathrm{Mb}$ are, presently, the most common types of deletions. Other researchers also reported one case in which 2-Mb fragments of the NF1 gene was absent; this fragment was absent mainly in the paternal chromosome. The patient with deleted type of mutations may show disease manifestations at a relatively early age and will have malformations and mental retardation [5].

In this study, we detected point mutation in the exonic region of the $N F 1$ gene in four type I nerve fibroma patients and one normal healthy subject in a family by using DNA direct sequencing and cloning techniques. We found a new SNP in the NF1 exon, which was located on the third codon with an unaltered amino acid sequence encoding for synonymous mutated exons, such as exons 7, 38, 50, and 56 . Cloning and sequencing confirmed that the mutation in exon 19 is CAG homozygous, and that in other samples is C/TAG heterozygous. The presence of the terminal codon led to degradation of the NF1 mRNA under the NMD mechanism, which in turn resulted in a unique copy of the NF1 gene encoding for the normal NF1 protein. We believe this to probably be the first report on NF1 mutation, and our data will supplement genetic and clinical databases and further enrich NF1 pathogenic genetic mutation map. Our study has provided new data on patients with NF1 and will help understand the genetic mechanism underlying this condition. Our data will also provide a basis for future investigations involving NF1 genetic screening and subsequent diagnosis and treatment.

Acknowledgements This work was financially supported by Grant No. 2011CB504302 from the 973 of China, Grant No.30940087 and No. 30872318 from the National Natural Science Foundation of China. We appreciate the valuable comments from other members of our laboratories.

Conflict of interest The authors declare that they have no conflict of interest.
Open Access This article is distributed under the terms of the Creative Commons Attribution Noncommercial License which permits any noncommercial use, distribution, and reproduction in any medium, provided the original author(s) and source are credited.

\section{References}

1. Ars E, Serra E, Garcia J, Kruyer H, Gaona A, Lazaro C, Estivill X (2000) Mutations affecting mRNA splicing are the most common molecular defects in patients with neurofibromatosis type 1. Hum Mol Genet 9(2):237-247. doi:ddd023

2. Gu W, Zhang F, Lupski JR (2008) Mechanisms for human genomic rearrangements. Pathogenetics 1(1):4. doi:10.1186/1755-8417-1-4

3. Huson SM, Harper PS, Compston DA (1988) Von Recklinghausen neurofibromatosis. A clinical and population study in south-east Wales. Brain 111(Pt 6):1355-1381

4. Kang H, Watkins G, Parr C, Douglas-Jones A, Mansel RE, Jiang WG (2005) Stromal cell derived factor-1: its influence on invasiveness and migration of breast cancer cells in vitro, and its association with prognosis and survival in human breast cancer. Breast Cancer Res 7(4):R402-410. doi:10.1186/bcr1022

5. Kehrer-Sawatzki H, Kluwe L, Funsterer C, Mautner VF (2005) Extensively high load of internal tumors determined by whole body MRI scanning in a patient with neurofibromatosis type 1 and a nonLCR-mediated 2-Mb deletion in 17q11.2. Hum Genet 116(6):466475. doi:10.1007/s00439-005-1265-4

6. Messiaen LM, Callens T, Roux KJ, Mortier GR, De Paepe A, Abramowicz M, Pericak-Vance MA, Vance JM, Wallace MR (1999) Exon 10b of the NF1 gene represents a mutational hotspot and harbors a recurrent missense mutation Y489C associated with aberrant splicing. Genet Med 1(6):248-253

7. Pasmant E, Sabbagh A, Masliah-Planchon J, Haddad V, Hamel MJ, Laurendeau I, Soulier J, Parfait B, Wolkenstein P, Bieche I, Vidaud M, Vidaud D (2009) Detection and characterization of NF1 microdeletions by custom high resolution array CGH. J Mol Diagn 11 (6):524-529. doi:10.2353/jmoldx.2009.090064

8. Praxedes LA, Pereira FM, Mazzeu JF, Costa SS, Bertola DR, Kim CA, Vianna-Morgante AM, Otto PA (2010) An illustrative case of neurofibromatosis type 1 and NF1 microdeletion. Mol Syndromol 1 (3):133-135. doi:10.1159/000319976319976

9. Thomson SA, Fishbein L, Wallace MR (2002) NF1 mutations and molecular testing. J Child Neurol 17(8):555-561, discussion 571$552,646-551$ 